\title{
Promotion Strategy of Classic Reading in Library under Network Environment
}

\author{
Jiliang Wang \\ Weifang University Library, Weifang 261061, China \\ wjl_wfu@163.com
}

Keywords: Library; Classic Reading; Reading Promotion

Abstract: On the basis of analyzing the environment of classical reading and readers' reading psychology, this paper discusses how to carry out classical reading promotion service under the network environment. The promotion of classical reading under the network environment must optimize the reading environment, establish a special organization for reading promotion, and expand the network promotion channels.

\section{Introduction}

Classics refer to works of exemplary and authoritative nature, usually those that have enduring vitality, have been tested by history, have important and lasting influence, and their contents are generally accepted by the public, or are of exemplary and authoritative nature in a certain professional field, and publish the most basic and root causes in this field. Knowledge [1]. Classics include not only collections of works with time span deposited by historical raccoons, but also works of values with popular culture under the influence of modern social ideology, which are constantly passed down and evolved, and which can arouse people to yearn for the pursuit of "truth, goodness and beauty" to enlighten human nature. The enduring charm of classics and the inexhaustible tension of classics determine that classical reading is different from practical and utilitarian reading and fashion reading in the context of popular culture. The epochal nature of classical reading calls for the cultivation of modern people with independent thinking and free critical thinking, so as to get rid of unconscious acceptance, weaken utilitarianism, improve moral and value awareness, meet people's needs for knowledge and their own development, and achieve people's ideal value.

\section{Information environment analysis of current classic reading}

\subsection{Networking of reading environment}

At present, the entire mankind is being swept up by an unprecedented cultural storm, this is the network culture. The reading environment of network culture relies on the computer network and takes the digital information as the main body. Because of its brand-new form of communication, huge amount of information and time-limited advantages, it has exerted a great influence and infiltration on College Students'life and learning. It has caused a serious impact and challenge to 
traditional culture and classical reading, and constantly impacted people. Reading and thinking. Due to the popularity of the Internet and the development of digital information technology, the reading rate and volume of books are declining, the number of books collected and purchased is sluggish, the number of Library borrowings is declining, the number of books read and online reading is changing, and the number of groups reading in a variety of ways and close to book reading is declining. In the network reading environment, people's reading interest and content are becoming more and more diversified and superficial, which makes the concept of time limitation fade away and the extension of time invisibly expand. In addition, in the network reading environment, the irreconcilable contradiction between different forms of media and readers'reading methods exists. The influence of this makes the understanding, importance and understanding of classics decrease.

\subsection{Digitalization of reading mode}

According to a survey conducted by China Press and Publication Research Institute, the reading rate of adult books, newspapers and periodicals in China was 58.0\%, 55.1\% and 40.3\% in 2014 . The fastest reading rate for digital books was 58.1\%, up 8 percentage points from $50.1 \%$ in 2013 . Digital reading has become the mainstream reading mode, including online reading, mobile phone reading, e-reader reading, CD-ROM reading, pad / PDA / MP4 / MP5 reading and so on. In 2014, $49.4 \%$ of adults read online, up 5.0 percentage points from $44.4 \%$ in 2013 ; $51.8 \%$ read on mobile phones, up 9.9 percentage points from $41.9 \%$ in 2013; 2.0\% read on CD-ROM, up 1.1 percentage points from $0.9 \%$ in 2013; 5.3\% read electronically. Read on the device, down 0.5 percentage points from 5.8\% in 2013; 9.9\% of adults use Pad (tablet computer) for digital reading; 34.4\% of adults use Wechat. [3] these statistics show that the way of reading carriers in China is becoming more and more digitalized.

\subsection{Fragmentation of reading content}

In the era of screen reading under the network environment, the content of reading is fragmented. Mobile social media such as micro-blogs and micro-letters cut the reader's spare time into pieces, while reading classics takes a long time, which makes it difficult for readers to read classics. Many readers do want to read more classic works of books, but they can't afford a lot of time. In fact, it is not that there is no time, but the time has been cut to pieces by mobile phone and other Internet media tools. Moreover, because of the convenience of information acquisition in the era of screen reading, its various search engines and digital tools make readers read classics into fragmented reading. Classic works are cut into chapters or paragraphs, and can be found and retrieved directly through a search engine without having to read them from beginning to end. We need to quote the point of view, the reader does not need to read the entire classic, but to use digital tools to achieve.

\section{The promotion strategy of Library Classics Reading under the network environment}

\subsection{Optimize reading promotion environment}

\subsubsection{Create comfortable reading space environment}

Libraries should be people-oriented, through the creation of a comfortable humanized reading environment, so that readers in the spirit of close to classical books. The reading environment is neat and bright, the furnishings are comfortable and elegant, making people feel kind and pleasant, giving people a feeling like bathing in the spring breeze, which is not only a kind of enjoyment, but also be nurtured unconsciously. [4] The library should adopt the open-shelf mode. When the readers 
enter the library with the borrowing card, they will enter a large reading room. All the reading rooms in the library are open and unimpeded. There is no independent reading room in concept. The readers can borrow, study, research and discuss freely in this library. In terms of function layout, facilities arrangement and internal and external environment construction, the library fully considers readers' wishes and habits, facilitates readers to use literature information, surrounds by indoor green plants, and gives readers kindness and care everywhere. Small area leisure discussion areas should be set up at suitable locations for students to freely exchange and discuss. Specialized research laboratories are set up for senior researchers, equipped with various learning and research tools for information retrieval and utilization, such as various application software, high-end workstations and printers, multimedia equipment, etc.

\subsubsection{Establish a sound reading resource environment}

Resources are the most basic material resources in the library's reading promotion service space. Without reading resources reading services will become the source of nothing. Therefore, building a rich and perfect reading resources environment system is the key to library's reading promotion service. Reading resources include not only traditional paper books but also various kinds of electricity. Sub literature. We must construct a convenient, fast and sharing reading resources environment, so that users can remote search and online reading library electronic resources and network resources, to achieve barrier-free communication between users and information resources. Reading resources environment is a wide range of multi-type, multi-system, heterogeneous resources sharing system, to cover the reading resources of different industry systems, to allow the producers, transmitters, operators, users of reading resources to participate in this space, especially to strengthen the union with book publishers. [5] Libraries should not only select and purchase high-quality classical reading resources on the basis of widely listening to experts'opinions, but also make use of their own unique resources collection and processing advantages, widely collect excellent teaching resources of teachers in schools, establish a navigation platform of teaching digital resources, and provide excellent online learning for college students. The construction of reading resources and environment should not be limited to the library, but should be connected with different resource systems extensively. We should adopt the idea of decentralized storage of resources, centralized catalogue retrieval, and providing services in different places. Full-text resources are distributed in different collection units, secondary documents are centralized in the sharing center, and resources are provided in different places. Units are provided separately. [6]

\subsection{Establish specialized reading guidance institutions and organizations}

\subsubsection{Set up a speciall reading guidance organization}

As a special institution for the collection and utilization of classical literature, the library has the responsibility to set up a special reading guidance institution to scientifically analyze and guide readers'reading behavior. For example, through the establishment of psychological growth reading room to guide the healthy reading of College students, to carry out "reading therapy" services, to help college students grow up healthy. Reading therapy is an assistant method of psychotherapy. The process of its service begins with librarians'understanding of readers' physical and mental development needs and their psychological problems and suggestions for appropriate reading materials. In the process of reading, the reader refers to the role of the material to solve the problem, comparing their own difficulties, so as to find a suitable solution for the individual [7]. Set up a team of students' psychological growth and carry out the practice of self-regulation of students' mental health. In-depth research and services on "reading therapy" should be carried out. Teachers 
recommend lists of books for spiritual growth to students, or good books for spiritual nourishment to students, so as to promote the healthy development of body and mind in the process of mutual communication. In addition, the psychological growth reading room also holds psychological Lovers'salon and spiritual growth lectures on a regular basis. Psychologists and moral education experts are invited to participate in student growth counseling to guide the healthy growth of College students. A psychological self-testing and experiencing center has been built in the electronic reading room, which provides experiential service for students' psychological growth.

\subsubsection{Innovation Library Reading Promotion Organization}

Besides good reading environment and scientific guiding mechanism, reading promotion also needs active reading promotion organization. For this reason, libraries can set up new reading promotion organizations, such as book drifting center, student apartment reading room, community reading room, etc., to push classic books to students and citizens. In order to stimulate readers' interest in reading, promote the exchange and sharing of classics, and achieve interactive reading. The Library of Tianfu College of Yunnan University of Finance and Economics has set up a special shelf in the hall of the library to set up a Book drifting center and a mobile Book drifting station in the dining hall and teaching building. In the past two years, the book drifting center of Tianfu College Library of Yunnan University of Finance and Economics has collected more than 1000 books, sorted out more than 800 books and read more than 3000 books, which has exerted a wide influence on College students. At the same time, the library of Tianfu College of Yunnan University of Finance and Economics, in line with the principle of "reader first", has set up a reading room for students'apartments, which runs the library around the readers. With the strong support of the Student Office Apartment Center, and in accordance with the principle of students'self-selection, self-management and self-use, students go to the library to select their favorite books, a student apartment reading room has been set up in the student apartment pilot project to recruit students to volunteer librarians for management. This not only extends the library's physical space, but also extends library services, more effectively.

\subsubsection{Strengthen sharing and cooperation in reading promotion}

Sharing and cooperation has become an important concept in library construction. Cross industry and cross regional library sharing alliances are becoming more sophisticated. Interlibrary loan, document transmission, joint editing and joint purchasing have become a reality in the library circle and have developed rapidly. All these laid the foundation for the cooperation and sharing of reading promotion. For the sustainable development of library reading promotion, we must take the road of cooperation and sharing, and establish a multi-directional, multi-level and multi-form reading promotion system. Reading promotion and sharing cooperation should include three levels: first, cooperation between libraries and schools, that is, University Libraries and departments within the school jointly carry out reading promotion activities. The second is the cooperation between libraries and societies, that is, to make full use of social forces, to co-operate with enterprises, bodies and other social organizations to hold reading activities. Third, inter-library cooperation, that is, through the establishment of regional or other cooperative alliances to jointly implement the promotion of reading.

\subsection{Expand online reading promotion channels}

\subsubsection{Actively strengthening the construction of reading promotion website}

The website construction of reading promotion website is the key to do a good job of reading 
promotion in the environment. First of all, we should set up a unified reading promotion column in the reading promotion website, open up an interactive exchange area and recommendation column for readers, set up a column for readers'online book review and classical reading guide, set up a navigation system for classical reading resources, and carry out the characteristics of classical reading resources mining, intelligent push and personalized customization. Secondly, we should carry out the classical reading promotion activities with the help of WEB technology, strengthen the interaction with readers through the network platform, and mutual check with Douban, and build an OPAC catalogue system that can automatically recommend books to readers, so as to realize one-stop search of massive reading resources and make readers'reading search more convenient. [8]

\subsubsection{Actively promotes Mobile Reading Promotion Service}

Nowadays, with the increasing number of Internet users, mobile phones have become a new terminal for reading, and mobile library services have become a new growth point for future reading. Therefore, it is necessary for libraries to build mobile digital libraries, carry out mobile reading promotion services, so as to facilitate readers to query, reserve, renew books and periodicals on mobile devices, as well as full-text literature information retrieval and query. In order to fully understand the readers'experience in classical reading, the library of Tianfu College of Yunnan University of Finance and Economics has created a group of classical reading micro-blog micro-letters. According to the characteristics of readers' classical reading tendency, we recommend the classical bibliography with strong readability and high academic value to micro-blog, discuss the readers'reading experience and attract readers to participate in it. Read the classic micro-blog exchange. At the same time, book reviewers, scholars and other experts were invited to participate in different reading groups to provide reading guidance and classical reviews for reading enthusiasts. The microblog classic reading book review group is mainly a reading exchange platform for reading classics, recommending classics and reviewing classics. Through this platform, librarians can timely understand the opinions of readers, adjust the strategy of classical reading promotion service, timely understand the level of library reading promotion service, and constantly improve the quality of classical reading promotion service. [9]

\subsubsection{Adapt classic to online reading}

The dependence on mobile micro-reading devices in the network environment has made reading no longer a simple way for readers to read text, but a human-computer interactive reading which combines reading means with reading content. It relies on virtual mobile reading environment and devices, and is a kind of multi-sensory reading. [10] Therefore, when promoting reading, it is necessary to make the presentation state of classical texts adapt to network reading, provide services in various forms besides classical content, and realize effective communication and connection between readers and classics, readers and readers, readers and authors. Using micro-signals and other network media to launch appropriate daily reading volume, design and arrange miniaturized and fragmented classical text suitable for network reading, alleviate readers'reading pressure, eliminate reading obstacles, and create a form of network reading for classical content. Due to the low tolerance of readers to monotony and dullness under the condition of mobile micro-reading on the internet, large paragraphs of the original text can be segmented and left blank properly under the premise of ensuring the integrity of the original text, so as to reduce the pressure of readers to read long classics. The obscure part provides easy-to-understand illustrations and micro-links, or highlights the classic brilliant content in eye-catching forms such as pictures, or inserts interesting points such as topics related to the content, so as to extend attention and adapt to the cognitive load of readers in digital media. 


\section{Conclusion}

As the growth point and innovation point of University library's reader service, reading promotion has become the core work of the library. We must reflect on the problems existing in the practice of reading promotion activities, innovate the concept of reading promotion, reconstruct the working mechanism of reading promotion, reorganize the existing professional posts of libraries, incorporate the work of reading promotion into the regular work scope of libraries, and manage it scientifically and regularly. Under the guidance of the Reading Committee and through the cooperation of libraries and schools, cooperatives between libraries and societies, and inter-library cooperation, we can make use of the mobile reading environment on the Internet to promote the continuous transformation and innovation of reading promotion services.

\section{References}

[1] Xu Huan. Children's traditional classics reading promotion research [J]. book and intelligence.2011, (2): 7-10

[2] Zhang Xuejun. On College Students' classics reading [J]. library forum, 2009, (5): 31-33

[3] China Academy of Press and Publication. Interpretation of the 13th National Reading Survey [EB/OL]. [2017-02-10]. http://book.ifeng.com/a/20150504/14604_0.shtml

[4] Liu Junxia. The construction of interactive reading promotion system in University Libraries --

[5] Yuan Xilin. Research on the Model of Information Resources Co-construction and Sharing and Its Theoretical Basis

[J]. Library and Information Work, 2008 (9): 102-105

[6] Guo Haiming. Library spatial service under the concept of resource sharing [J]. Library theory and practice, 2011

(7): $1-4$

[7] Chen Shumei. Talking about the Reading Guidance Service of Public Libraries from the Present Situation of Reading Promotion Activities in Taiwan [J]. Library Construction, 2006, (5): 78-81

[8] Lei Chunyu. Probe into the Reading Promotion Work of University Libraries in the Network Era [J]. Management Informatization in China. 2017 (2): 175

[9] Zhang Lihua. Application of micro-book review in the promotion of classical reading in University Libraries [J]. Journal of Sichuan Library, 2016, (5): 87-89

[10] Luan Xuemei. The classic reading promotion strategy in the micro age. Library intelligence work, 2016 (18):

116-120 